\title{
Precision cosmology with a combination of wide and deep Sunyaev-Zeldovich cluster surveys
}

\author{
Satej Khedekar ${ }^{1}$, Subhabrata Majumdar ${ }^{1}$ and Sudeep Das ${ }^{2}$ \\ ${ }^{1}$ Tata Institute of Fundamental Research, Homi Bhabha Road, Colaba, Mumbai - 400076, India. \\ ${ }^{2}$ Berkeley Center for Cosmological Physics, LBL and Department of Physics, \\ University of California, Berkeley, California 94720, USA
}

(Dated: May 28, 2022)

\begin{abstract}
We show the advantages of a wedding cake design for Sunyaev-Zel'dovich cluster surveys. We show that by dividing up a cluster survey into a wide and a deep survey, one can essentially recover the cosmological information that would be diluted in a single survey of the same duration due to the uncertainties in our understanding of cluster physics. The parameter degeneracy directions of the deep and wide surveys are slightly different, and combining them breaks these degeneracies effectively. A variable depth survey with a few thousand clusters is as effective at constraining cosmological parameters as a single depth survey with a much larger cluster sample.
\end{abstract}

PACS numbers:

Introduction. - The abundance and redshift distribution $\frac{d N}{d z}$ of clusters are potentially important probes of cosmological parameters [1 5]. Recently, number counts from cluster surveys in optical (e.g RCS [7] and SDSS [8]) and microwave [9] have been used to constrain cosmology. In the coming decade, dedicated surveys in different wavebands (for example, ACT, DES, eROSITA, etc.) plan to use clusters as probes of precision cosmology. A correct cosmological interpretation of the observed $\frac{d N}{d z}$ depends on precise knowledge of the limiting mass of the survey at a given redshift. Unfortunately, the mass of a cluster is not directly observable, but has to be obtained through proxy observables such as X-ray surface brightness and temperature 10], Sunyaev Zeldovich (SZ) decrement [11, 12], cluster richness [13, 14] and lensing [15, 16]. Uncertainties in the observable to mass conversion create degeneracies between cosmology and cluster physics and degrade cosmological constraints. There have been a number of attempts in the literature to break such degeneracies, for example, through the so called 'self-calibration' techniques [5, 6, 17]. Other approaches include an 'unbiased' mass follow-up of a subsample of the survey clusters [17, 18] or better theoretical modeling of clusters to predict the form of mass-observable scaling relation [19 21]. All of these are equivalent to putting priors on the parameter space of the mass-observable relations. One can also try to optimize the cluster surveys so as to get the best possible survey yield 22].

In this Letter, we show that by simply dividing the total observation time between deep and wide surveys, parameter degeneracies can be effectively broken leading to tight cosmological constraints. This is without the need of any external information or a costly mass followup program. Throughout this paper, we assume a spatially flat cosmological model with a constant equation of state $w$ for dark energy with the fiducial cosmological parameters taken as $h=0.717, \Omega_{\mathrm{m}}=0.258, w=-1$, $\Omega_{\mathrm{b}}=0.044, n_{s}=0.963$ and $\sigma_{8}=0.796$ consistent with the WMAP 5-year results.

Preliminaries. - The redshift distribution of detectable clusters in a survey over an area $\Delta \Omega$ is,

$$
\frac{d N}{d z}(z)=\Delta \Omega \frac{d V}{d z d \Omega}(z) \int_{0}^{\infty} f\left(M \mid M_{\lim }(z)\right) \frac{d n(M, z)}{d M} d M
$$

where $\frac{d V}{d z d \Omega}$ is the comoving volume element and $\frac{d n}{d M}$ is the cluster mass function (taken from simulations by Jenkins et al. [23]). A mass $\operatorname{limit} M_{\lim }(z)$ is effectively imposed through the complementary error function $f\left(M \mid M_{\lim }(z)\right)$ [24], which is used to model a $20 \%$ logarithmic scatter in the mass-proxy relation centered about $M_{\text {lim }}$. Figure 1 shows the plots of $M_{\text {lim }}$ versus $z$ for various array sensitivities of the ACT survey along with the corresponding redshift distribution of clusters. For an SZ survey, the flux-mass scaling relation is used to determine $M_{\lim }(z)$ [17], $\operatorname{flux}_{\mathrm{sz}}(\mathrm{z}, \nu) d_{A}^{2}(z)=$ $2.699 \times 10^{11} g(\nu) A_{\mathrm{SZ}} M_{200}^{\alpha} E^{2 / 3}(z)(1+z)^{\gamma}$, where $g(\nu)$ is the frequency dependence of the SZ distortion, $M_{200}$ is in units of $M_{\odot}$ and $d_{A}$ is the angular diameter distance in units of Mpc. The Hubble expansion is parameterized as $H(z)=H_{0} E(z)$. The parameter $\gamma$ captures deviation in the redshift evolution of the cluster scaling relation from the self similar case, and we assume $\gamma \sim 0$. We choose the SZ scaling relations to be compatible with recent observations 21, 25] with $\log \left(A_{\mathrm{SZ}}\right)=-28.067$ and $\alpha$ $=1.612$.

Majumdar and Mohr 17, 18 have shown that a substantial improvement in the cosmological parameter constraints can be obtained by adding a follow-up of a fraction of the cluster sample to calibrate the massobservable relation through detailed observations in XRay, SZ or galaxy spectroscopy. We construct a mock follow-up survey with $\sim 100$ clusters having 'unbiased' mass measurements in the range $2-10 \times 10^{14} h^{-1} M_{\odot}$ between $0.3 \leq \mathrm{z} \leq 1.1$ with $20-40 \%$ error on masses. We use both Fisher matrix and MCMC techniques, details on which can be found in many references, e.g. 


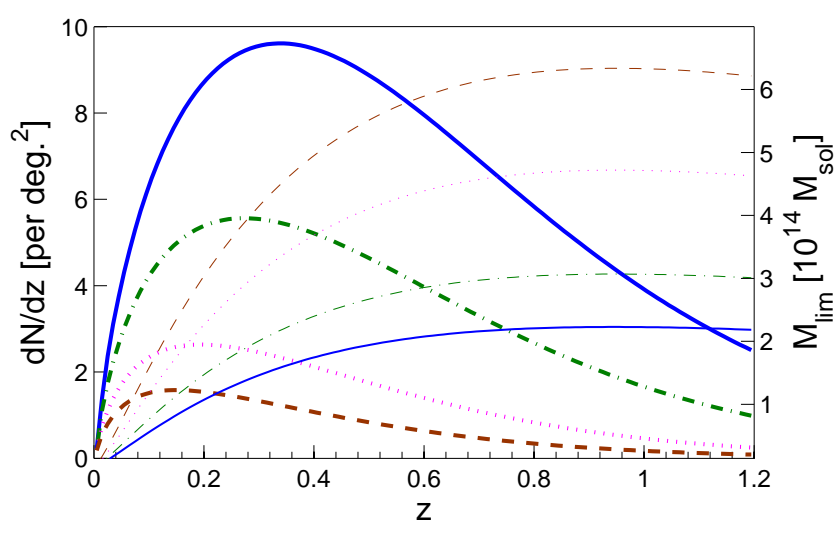

FIG. 1: The cluster redshift distribution $\frac{d N}{d z}$ (thick lines) and $M_{\text {lim }}$ (thin lines) vs redshift, for a SZ survey with array sensitivities of 3.54 (solid), 10.61 (dot-dashed), 21.21 (dotted) and 34.12 (dashed) $\mu \mathrm{K} \sqrt{\mathrm{s}}$.

[1, 4, 7, 17, 26, 27]. Full MCMC runs are made to estimate the covariance matrix between cosmological parameters and $\left(A_{S Z}, \alpha, \gamma\right)$ from follow-up. The full parameters space is spanned by $\left(h, n_{s}, \Omega_{\mathrm{b}}, \Omega_{m}, w, \sigma_{8}, A_{S Z}, \alpha, \gamma\right)$ and we use Gaussian priors with standard deviations of $0.027,0.015$ and 0.003 on the parameters $h, n_{s}$ and $\Omega_{\mathrm{b}}$ respectively.

We assume the survey instrument to be a telescope with an angular resolution of $\theta_{\text {fwhm }}$ with a bolomet-

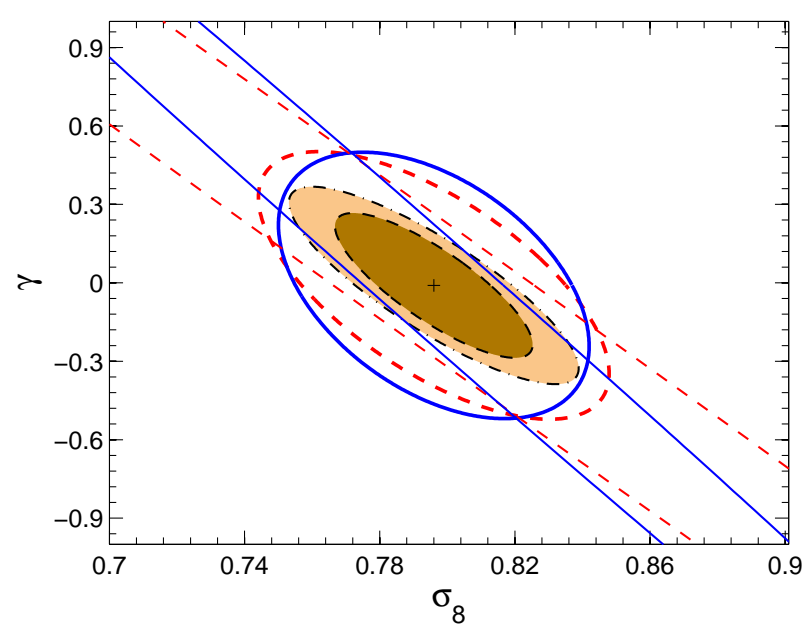

FIG. 2: Parameter degeneracy in the $\sigma_{8}-\gamma$ plane for a wedding cake survey with $f_{\text {time }}=0.75$ and $r=1 / 9$. The thick ellipses indicate constraints when mass follow-up is added, while the thin cigar-like ellipses are the constraints for unknown cluster evolution. For these, the dashed lines are the constraints from the small area survey, while the solid lines are constraints from the large area survey. The light (dark) filled ellipse shows the combined constraint from the two surveys with mass followup (unknown evolution). Note that, the intersection of the two cigars give the tighter constraints. ric detector array of equivalent array-sensitivity (noiseequivalent temperature) $\sigma_{a}$. For a survey of area $A$ and duration $t_{\mathrm{obs}}$, the time spent on each of the $N_{\text {pix }}=$ $A / \theta_{\text {fwhm }}^{2}$ sky-pixels is $t_{\text {pix }}=t_{\text {obs }} / N_{\text {pix }}$, giving a noise per sky-pixel of $\Delta_{T}=\sigma_{a} / \sqrt{t_{\text {pix }}}$. In particular, we assume a survey of area $A=2000$ sq. deg. at $150 \mathrm{GHz}$ with $\theta_{\text {fwhm }}=1^{\prime}$ and a duration of $10^{7}$ seconds. We consider three cases for the array-sensitivity - 3.54, 10.61 and $21.21 \mu \mathrm{K} \sqrt{\mathrm{s}}$. In perspective, for its 2008 season, the ACT had an array sensitivity of $\sim 34 \mu \mathrm{K} \sqrt{\mathrm{s}}$ at 148 GHz. These array sensitivities would correspond to flux limits of $2.7,8.3,16.6$ and 26.7 mJy respectively for a single area of $2000 \mathrm{sq}$. deg. in the ACT survey. The SPT has similar configurations. Given a thermal noise $\Delta_{T}$ per sky-pixel, the flux limit is computed as follows: we assume that the SZ decrement/increment has to be integrated over roughly 30 pixels to detect a cluster, so the $5 \sigma$ flux limit becomes $5 \sqrt{30} \Delta_{T}$ converted from $\mu \mathrm{K}$ into mJy through the derivative of the blackbody function [28]. However, flux sensitivity may be limited by the covariant noise from CMB as well as confusion arising from point sources; we use a fixed (irrespective of how deep we go on a patch) limiting flux value of $2.5 \mathrm{mJy}$ as an absolute lower cutoff.

\section{Results.-}

We illustrate the strength of a wedding cake survey by breaking the fiducial $2000 \mathrm{deg}^{2}$ survey area into two patches keeping the total observing time fixed. We use

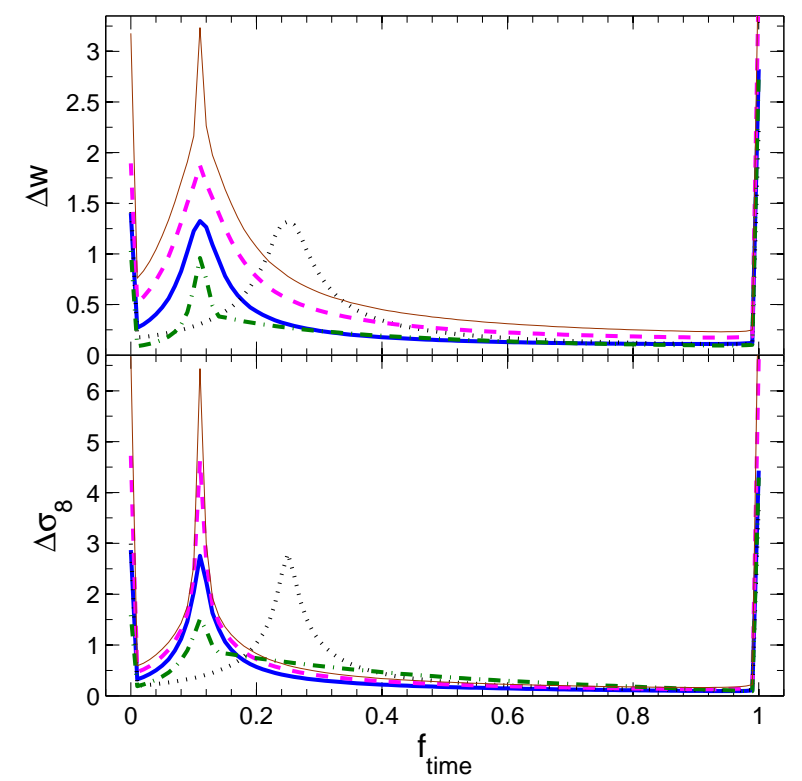

FIG. 3: Constraints on $w$ (upper panel), and $\sigma_{8}$ (lower panel) from only number count observations for a two component survey design as a function $f_{\text {time }}$. The curves are plotted for $\sigma_{a}(\mu \mathrm{K} \sqrt{\mathrm{s}})=3.53, \mathrm{r}=1 / 9$ (dot-dashed); $\sigma_{a}=10.61, \mathrm{r}=1 / 9$ (solid); $\sigma_{a}=21.21, \mathrm{r}=1 / 9$ (dashed), $\sigma_{a}=10.61 \& \mathrm{r}=1 / 4$ (dotted), and $\sigma_{a}=31.83 \& \mathrm{r}=1 / 9$ (thin). 
$f_{\text {time }}$ to denote the fraction of total time spent on the smaller area, and $r$ to denote the ratio of the smaller to the larger survey area. Varying $f_{\text {time }}$ amounts to different integration time on each patch and hence different flux limits. Different flux limits lead to changes in the shape of $\frac{d N}{d z}$ (see Figure 1), so that the two patches yield different parameter degeneracy directions. When the constraints are combined, the parameter degeneracies are effectively broken, leading to tight constraints on cosmological parameters. This is evident in Fig. 2 which illustrates how the notorious cosmology-cluster physics degeneracy (such as $\sigma_{8}-\gamma$ ) can be broken. A multiple depth survey is akin to having surveys with multiple limiting masses or $M_{\mathrm{lim}}$. This provides binning in both the observable mass proxy as well as redshift. The binning in the mass is what helps in breaking the degeneracies, by extracting out more information from the survey [24].

a) Wedding cake only. - The marginalized constraints on $w$ and $\sigma_{8}$ from an SZ survey like the ACT for various detector noise levels as a function of $f_{\text {time }}$ are shown in Fig. 3 It shows that having a single sky area to a fixed depth with no prior knowledge of cluster parameters gives no constraints on cosmology, in qualitative agreement with previous work [17]. However, the constraints dramatically improve as soon as the survey is broken up into wide and deep parts. For our survey of total duration $10^{7} \mathrm{~s}$ and with cluster yield $N_{\mathrm{cl}} \sim(3200,2700)$ for the wide and deep parts (for $f_{\text {time }} \sim 0.75, r=1 / 9$ and $\left.\sigma_{a}=3.54 \mu \mathrm{K} \sqrt{\mathrm{s}}\right)$ one can get $\left(\Delta \Omega_{m}, \Delta w, \Delta \sigma_{8}\right)=(0.105$, $0.115,0.116)$ just from $\frac{d N}{d z}$. For a similar exercise, a sin-

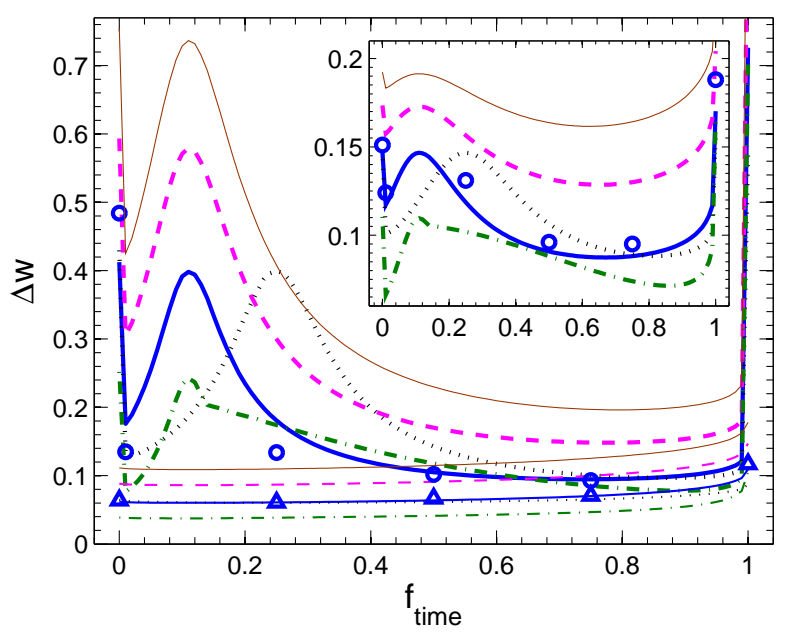

FIG. 4: Constraints on $w$ in a wedding cake survey. The main figure shows the constraints when $\left(A_{S Z}, \alpha\right)$ are known. The thick (thin) curves correspond to an unknown (known) cluster evolution respectively. The inset displays constraints when $\left(A_{S Z}, \alpha, \gamma\right)$ are free parameters but mass follow-up is added. The curves are plotted for $\sigma_{a}(\mu \mathrm{K} \sqrt{\mathrm{s}})=21.21, \mathrm{r}=$ $1 / 9$ (dashed); $\sigma_{a}=10.61, \mathrm{r}=1 / 9$ (solid); $\sigma_{a}=10.61, \mathrm{r}=$ $1 / 4$ (dot-dashed), and $\sigma_{a}=31.83 \& \mathrm{r}=1 / 9$ (thin). gle depth survey of $2000 \mathrm{deg}^{2}$ with $N_{c l} \sim 7500$ would get $\left(\Delta \Omega_{m}, \Delta w, \Delta \sigma_{8}\right)=(0.772,1.41,3.31) !$ Thus, moving to deep+wide survey acts like having an additional mass follow-up (or 'self-calibration') without any extra effort. Note that the bump at $f_{\text {time }}=0.1$ for $r=1 / 9$ corresponds to having the same flux limits for the two surveys such that the parameter degeneracies are the same and there is no improvement in the constraints. For a higher area ratio, $r=1 / 4$, the bump is shifted to the right, as expected.

b) Breaking degeneracies: Mass follow-up vs wedding cake survey. - Our lack of knowledge in the evolution in the mass-observable relations can lead to cosmological constraints getting weaker by factors of 4 or more [18], which can be restored through either a mass follow-up program or using cluster bias as a proxy for mass (i.e, self-calibration). However, the former requires a costly follow-up effort and 'unbiased' mass estimators, while for the latter, one needs to be careful about scale dependent bias. Here, we put forward a simple solution for this problem. Following [17], we parameterize the redshift evolution of mass - observable relation with a factor $(1+z)^{\gamma}$ in the scaling relation. To start, we assume that $A_{S Z}$ and $\alpha$ are known perfectly. Fig. 4 shows the constraints on $w$ when $\gamma$ is known/unkown. We find that a two component survey improves the constraints on $w$ by a factor of 4 for the case when cluster evolution is unknown. This is mainly by breaking the degeneracy existing between the parameters $\gamma$ and $\sigma_{8}$. As shown in Table [1 the constraints on $w$ improve from 0.4 to 0.095 when we have a two component survey. Note, that the results from MCMC (triangles and circles in Fig. 4) confirm the trends seen from the Fisher pipeline. The inset in Fig. 4 shows the constraints obtained on the parameter $w$ when both cluster structure and evolution is unknown but we have some information on all the three cluster parameters from mass follow-up, the currently preferred method for upcoming cluster surveys. The tightest constraints on $w$ are obtained when the smaller patch is observed between $70 \%$ and $95 \%$ of the total time, depending of the array-sensitivity. Adding follow-up observations to cluster counts already breaks the degeneracy in cosmologycluster physics, but a wedding cake design can still improve $w$ constraints by a factor of 1.5 or more. Again, we confirm the trend seen in Fisher matrix forecasts with MCMC simulations. A detailed comparison of Fisher-vsMCMC for cluster cosmology will be given in [29].

Discussion. - We have shown that by breaking up a cluster survey into a wide and a deep parts, tight cosmological constraints can be obtained without the need for costly mass follow-up observations or self-calibration, which may be limited by our understanding of scaledependent bias. For different scenarios - having only number counts, having a follow-up survey, or known scaling relations with unknown redshift evolution, we always find an improvement in the constraints on the dark en- 
TABLE I: Comparison of $1 \sigma$ parameter constraints from a cluster survey of array-sensitivity $10.61 \mu \mathrm{K} \sqrt{\mathrm{s}}$ and duration $10^{7}$ seconds. The boldface numbers with similar brackets are for easy comparison of a single component vs two component survey.

\begin{tabular}{|c|c|c|c|}
\hline \multicolumn{4}{|c|}{ Single area $\left(2000 \mathrm{deg}^{2}\right)$ survey } \\
\hline & unknown & unknown & with mass \\
\hline Parameter & scaling & redshift evolution & follow-up \\
\hline$\Delta \Omega_{m}$ & 0.676 & 0.019 & 0.032 \\
\hline$\Delta w$ & 1.343 & $(0.400)$ & {$[0.147]$} \\
\hline$\Delta \sigma_{8}$ & 2.849 & 0.121 & 0.028 \\
\hline \multicolumn{4}{|c|}{ Wedding cake $\left(1800+200 \mathrm{deg}^{2}\right)$ survey } \\
\hline & unknown & unknown & with mass \\
\hline Parameter & scaling & redshift evolution & follow-up \\
\hline$\Delta \Omega_{m}$ & 0.105 & 0.009 & 0.030 \\
\hline$\Delta w$ & {$[0.115]$} & $(0.095)$ & 0.088 \\
\hline$\Delta \sigma_{8}$ & 0.116 & 0.019 & 0.028 \\
\hline
\end{tabular}

ergy equation of state $w$. Our finding are summarized in Table I which clearly shows that a two component survey is always superior to a single area survey. For an SZ survey like the ACT, just number count observations with the two component design are capable of constraining cosmology very well $\left(\Delta \Omega_{m}=0.105, \Delta w=\right.$ 0.115 and $\left.\Delta \sigma_{8}=0.116\right)$, when a single area approach would produce cosmologically uninteresting constraints. These results can be further improved by having followup observations of $\sim 100$ clusters. A wedding cake survey is also able to effectively break the degeneracy between non-standard evolution $\gamma$ and $\sigma_{8}$, and provide factors of several improvements in constraints over a single area survey (third column of Table \).

With the advent of a large number of surveys in the near future, we should be able to use clusters as independent probes of cosmology. Precision cluster cosmology is traditionally thought to require either a good understanding of cluster physics, or detailed mass follow-up observations of a fraction of the detected clusters. We show that having a wedding cake survey strategy is an effective way to beat down cosmological parameter degeneracies. Our work provides an important new insight into developing future SZ survey designs.

The authors would like to thank David Spergel for discussions. SM also thanks Anya Chaudhuri, Christoph Pfrommer and Joe Mohr for many discussions on scaling relations over the years.

[1] G. Holder, Z. Haiman, and J. J. Mohr, ApJ 560, L111 (2001), arXiv:astro-ph/0105396.

[2] L. Wang and P. J. Steinhardt, ApJ 508, 483 (1998), arXiv:astro-ph/9804015.

[3] E. S. Levine, A. E. Schulz, and M. White, ApJ 577, 569 (2002), arXiv:astro-ph/0204273.

[4] J. Weller, R. A. Battye, and R. Kneissl, Physical Review Letters 88, 231301 (2002), arXiv:astro-ph/0110353.
[5] W. Hu, Phys. Rev. D 67, 081304(R) (2003), arXiv:astroph/0301416.

[6] M. Lima and W. Hu, Phys. Rev. D 70, 043504 (2004), arXiv:astro-ph/0401559.

[7] M. D. Gladders, H. K. C. Yee, S. Majumdar, L. F. Barrientos, H. Hoekstra, P. B. Hall, and L. Infante, ApJ 655, 128 (2007), arXiv:astro-ph/0603588.

[8] E. Rozo, R. H. Wechsler, B. P. Koester, T. A. McKay, A. E. Evrard, D. Johnston, E. S. Sheldon, J. Annis, and J. A. Frieman, ArXiv Astrophysics e-prints (2007), arXiv:astro-ph/0703571.

[9] K. Vanderlinde et al., ArXiv Astrophysics e-prints (2010), arXiv:astro-ph/1003.0003.

[10] H. Ebeling, A. C. Edge, S. W. Allen, C. S. Crawford, A. C. Fabian, and J. P. Huchra, MNRAS 318, 333 (2000), arXiv:astro-ph/0003191; H. Ebeling, A. C. Edge, and J. P. Henry, ApJ 553, 668 (2001), arXiv:astroph/0009101; A. Vikhlinin, B. R. McNamara, W. Forman, C. Jones, H. Quintana, and A. Hornstrup, ApJ 502, 558 (1998), arXiv:astro-ph/9803099; H. Böhringer, P. Schuecker, L. Guzzo, C. A. Collins, W. Voges, R. G. Cruddace, A. Ortiz-Gil, G. Chincarini, S. De Grandi, A. C. Edge, et al., A\&A 425, 367 (2004), arXiv:astro$\mathrm{ph} / 0405546$.

[11] Z. Staniszewski et al., ApJ 701, 32 (2009), 0810.1578.

[12] A. D. Hincks et al., ArXiv e-prints (2009), 0907.0461.

[13] M. Postman, L. M. Lubin, J. E. Gunn, J. B. Oke, J. G. Hoessel, D. P. Schneider, and J. A. Christensen, AJ 111, 615 (1996), arXiv:astro-ph/9511011.

[14] B. P. Koester et al., ApJ 660, 239 (2007), arXiv:astro$\mathrm{ph} / 0701265$.

[15] D. Wittman, I. P. Dell'Antonio, J. P. Hughes, V. E. Margoniner, J. A. Tyson, J. G. Cohen, and D. Norman, ApJ 643, 128 (2006), arXiv:astro-ph/0507606.

[16] Zitrin, A., Broadhurst, T., Rephaeli, Y., \& Sadeh, S. 2009, arXiv:0907.4232

[17] S. Majumdar and J. J. Mohr, ApJ 613, 41 (2004), arXiv:astro-ph/0305341.

[18] S. Majumdar and J. J. Mohr, ApJ 585, 603 (2003), arXiv:astro-ph/0208002.

[19] J. D. Younger, Z. Haiman, G. L. Bryan, and S. Wang, ApJ 653, 27 (2006), arXiv:astro-ph/0605204.

[20] B. A. Reid and D. N. Spergel, ApJ 651, 643 (2006), arXiv:astro-ph/0601133.

[21] A. Chaudhury and S. Majumdar, In preparation.

[22] R. A. Battye and J. Weller, MNRAS 362, 171 (2005), arXiv:astro-ph/0410392.

[23] A. Jenkins, C. S. Frenk, S. D. M. White, J. M. Colberg, S. Cole, A. E. Evrard, H. M. P. Couchman, and N. Yoshida, MNRAS 321, 372 (2001), arXiv:astro$\mathrm{ph} / 0005260$.

[24] M. Lima and W. Hu, Phys. Rev. D 72, 043006 (2005), arXiv:astro-ph/0503363.

[25] M. Bonamente, M. Joy, S. J. LaRoque, J. E. Carlstrom, D. Nagai, and D. P. Marrone, ApJ 675, 106 (2008), 0708.0815 .

[26] M. Tegmark, A. N. Taylor, and A. F. Heavens, ApJ 480, 22 (1997), arXiv:astro-ph/9603021.

[27] A. Lewis and S. Bridle, Phys. Rev. D 66, 103511 (2002), arXiv:astro-ph/0205436.

[28] M. White and S. Majumdar, ApJ 602, 565 (2004), arXiv:astro-ph/0308464.

[29] S. Khedekar and S. Majumdar, In preparation. 